\title{
Numerical Study for the Pressure Transient Behavior of Fractured Shale Gas Reservoirs Using a Dual-Mechanism Dual-Porosity Model
}

\author{
Xiaozhou Zhang ${ }^{1, a^{*}}$ and Changqian Zhu ${ }^{1, b}$ \\ ${ }^{1}$ China University of Petroleum (Beijing), Beijing, China \\ azhangxiaozhou113@gmail.com, b804570100@qq.com
}

Keywords: Shale Gas Reservoirs; Numerical Simulation; Desorption; Stress Sensitivity; Dual-Porosity; Pressure Transient.

Abstract. Shale gas reservoirs have become the focus of considerable attention as primary energy resource over the past decades worldwide. Numerical modeling technique plays a critical role in providing the essential tools for evaluating and managing the development of such complex systems. Gas flow in shale formation involves substantial nonlinear complexity. The flow behavior may be governed by the mechanisms of desorption and rock unconsolidation within stimulated natural fracture network. It is very likely that the fracture network is unpropped or weakly propped after hydraulic fracturing. Desorption is also an important mechanism which contributes to the gas production. In this work, a dual-mechanism dual-porosity model was established for pressure transient analysis. Finite-difference method was used for discretizing fluid equations, and Newton method was used to solve the algebraic equations. The typical curves in terms of dimensionless pseudopressure and time were drawn with different sets of parameters. The numerical well-testing model could provide theoretical basis for interpreting production data of shale gas reservoirs.

\section{Introduction}

Shale gas resources are presently received great attention because of their potential to supply the world with enormous amount of energy. Even though significant progresses has been made towards commercial development of gas and oil from unconventional resources in the past few years, characterization of gas flow in theses reservoirs remain a challenge [1]. This is primarily because there exist so many nonlinear processes, stress sensitivity effect, adsorption/desorption as well as severe heterogeneity on various scales of fractures [2]. It has been shown that conductive and interconnected natural fractures can substantially influence well performance [3]. Therefore transient pressure analysis for unconventional gas reservoirs cannot rely entirely on traditional, analytical based approaches and have to be carried out by numerical approach in general.

\section{The Physical and Mathematical Model}

A radial dual-porosity model of vertical well in naturally fractured shale gas reservoir was established by taking into account the effect of pressure-dependent permeability and adsorption/desorption. The physical model is shown in fig. 1. The effects of gravity and capillary pressure are neglected, and single-phase gas flow is considered.

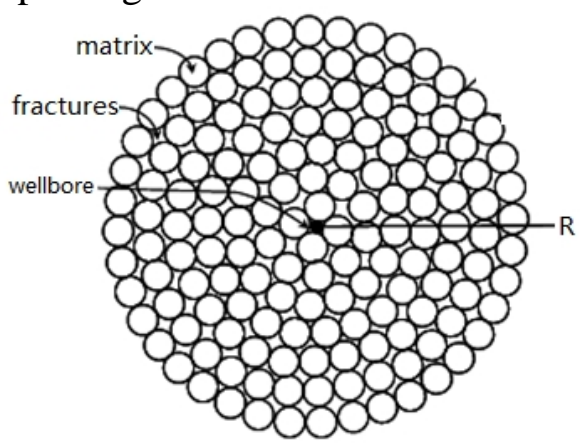

Fig.1 Physical model of vertical well in dual-porosity reservoir 
Adsorption. Natural gas or methane molecules are adsorbed mainly to the carbon-rich components, i.e. kerogen. In our model, the mass of adsorbed gas in formation volume $\mathrm{V}$ is described using the Langmuir isotherm in terms of pseudopressure [4]:

$V_{E}=\frac{V_{L} \varphi(p)}{\varphi\left(p_{L}\right)+\varphi(p)}$

Where $V_{L}$ is Langmuir volume, $\mathrm{m}^{3} / t ; V_{E}$ is volume of adsorption, $\mathrm{m}^{3} / t$ and $p_{L}$ is Langmuir pressure, $M P a$.

Stress Sensitivtity. In unconventional shale formations with nano-size pores, geomechanics effect can be relatively large and may have a significant impact on fractures' permeability. Wang et al. Show that permeability in the Marcellus Shale is pressure dependent and decreases with an increase in confining of pore pressure [5]. The method used here to account this effect in the numerical model is shown as below:

$k(\varphi(p))=k_{f i} \exp \left[-\beta\left(\varphi\left(p_{i}\right)-\varphi(p)\right]\right.$

Where $k_{f i}$ is initial permeability of fractures, $10^{-3} \mu m^{2} ; \beta$ is the stress-sensitive coefficient, $m P a . s / M P a^{2}$.

Flow Model. Mass conservation equation in pseudo-steady state:

Fracture: $\nabla\left(\frac{k_{f}}{\mu_{g}} \rho_{g f} \nabla p_{g f}\right)=\frac{\partial}{\partial t}\left(\rho_{g f} \phi_{f}\right)+\frac{\alpha \rho_{g m} k_{m}}{\mu_{g}}\left(p_{f}-p_{m}\right)$

Matrix: $\frac{\alpha \rho_{g m} k_{m}}{\mu_{g}}\left(p_{f}-p_{m}\right)=\frac{\partial}{\partial t}\left(\rho_{g m} \phi_{m}+\rho_{g s c} V_{E}\right)$

Using equations (2), (3) for considering adsorption and pressure-dependent permeability.

With the integration of equations (1)-(4), we can derive:

$\nabla\left(\frac{k_{f} p_{g f}}{\mu_{g} z_{g}} \nabla p_{g f}\right)=\frac{\phi_{f} p_{g f} c_{f}}{z_{g}}\left(\frac{\partial \Delta p_{g m}}{\partial t}\right)+\frac{\phi_{m} p_{g m}}{z_{g}}\left[c_{m}+\frac{\rho_{g s c} V_{L} \varphi\left(p_{L}\right)}{\rho_{g m} \phi_{m}\left(\varphi\left(p_{L}\right)+\varphi\left(\bar{p}_{g m}\right)\right)}\right]\left(\frac{\partial \Delta p_{g m}}{\partial t}\right)$

We can also define:

Matrix total compressibility: $\bar{c}_{t m}=c_{t m}+\frac{\rho_{g s c} V_{L} \varphi\left(p_{L}\right)}{\rho_{g m} \phi_{m}\left(\varphi\left(p_{L}\right)+\varphi\left(\bar{p}_{g m}\right)\right)}$

Interporosity flow coefficient: $\lambda=\alpha r_{w}{ }^{2} \frac{k_{m}}{k_{f i}}$

The storativity ratio: $\omega=\frac{\left(\phi c_{t}\right)_{f}}{\left(\phi c_{t}\right)_{f}+\left(\phi \bar{c}_{t}\right)_{m}}$

Pseudopressure: $\varphi_{\partial}=2 \int_{p_{0}}^{p} \frac{p}{\mu Z} d p(\partial=m$ or $f)$

The dimensionless pseudopressure: $\varphi_{D \partial}=\frac{\pi k_{f i} h_{f t} T_{s c}}{q_{s c} p_{s c} T} \Delta \varphi_{\partial}(\partial=m$ or $f)$

The dimensionless time: $t_{D}=\frac{k_{f i}}{\left(\phi_{m} \bar{c}_{t m} \mu_{g}+\phi_{f} c_{t f} \mu_{g}\right) r_{w}{ }^{2}} t(\partial=m$ or $f)$

The dimensionless radius: $r_{D}=\frac{r}{r_{w}}, r_{D m}=\frac{r_{m}}{r_{w}}$

The dimensionless stress-sensitive coefficient: $\beta_{D}=\frac{q_{s c} p_{s c} T \beta}{\pi k_{f i} h_{f t} T_{s c}}$

Transform the model's equations in dimentionless terms, and define:

$\varphi_{D f}=-\frac{1}{\beta_{D}} \ln \left(1-\beta_{D} \eta\right), \varphi_{D m}=\xi$ 
Then we can derive: $\frac{\partial^{2} \eta}{\partial r_{D}{ }^{2}}+\frac{1}{r_{D}}\left(\frac{\partial \eta}{\partial r_{D}}\right)=\left(\frac{w}{1-\beta_{D} \eta} \frac{\partial \eta}{\partial t_{D}}+(1-w) \frac{\partial \xi}{\partial t_{D}}\right)$

$$
(1-w) \frac{\partial \xi}{\partial t_{D}}=-\lambda\left(\frac{1}{\beta_{D}} \ln \left(1-\beta_{D} \eta\right)+\xi\right)
$$

Initial condition: $\eta\left(r_{D}, t_{D}=0\right)=0$

Inner boundary condition: $\left\{C_{D}\left[\frac{1}{1-\beta_{D} \eta} \frac{\partial \eta}{\partial t_{D}}-S \frac{\partial\left(r_{D} \frac{\partial \eta}{\partial r_{D}}\right)}{\partial t_{D}}\right]-\left(r_{D} \frac{\partial \eta}{\partial r_{D}}\right)\right\}_{r_{D}=1}=1$

External boundary condition: $\lim _{r_{D} \rightarrow \infty} \eta\left(r_{D}, t_{D}\right)=0$

\section{Numerical Formulation}

The numerical approach of this work uses finite-difference method for discretizing fluid equations, and apply Newton method for solving the resulting nonlinear sets of algebraic equations.

Discrete Equations. For the convinent of computation, we first apply coordinate conversion: $r_{D}=e^{(i-1) \Delta x}$, where $\Delta x=\ln r_{D} /(N-1)$, and $\mathrm{N}$ is the number of gridblocks.

Then discretize equations (15)-(19) in finite-difference scheme:

$$
\begin{aligned}
& \frac{\eta_{i+1}{ }^{n+1}-2 \eta_{i}^{n+1}+\eta_{i-1}{ }^{n+1}}{\Delta x^{2}}=\left(\frac{w}{1-\beta_{D} \eta_{i}{ }^{n+1}} \frac{\eta_{i}^{n+1}-\eta_{i}{ }^{n}}{\Delta t}+(1-w) \frac{\xi_{i}^{n+1}-\xi_{i}{ }^{n}}{\Delta t}\right) e^{2(i-1) \Delta x} \\
& (1-w) \frac{\xi_{i}^{n+1}-\xi_{i}^{n}}{\Delta t}=-\lambda\left(\frac{1}{\beta_{D}} \ln \left(1-\beta_{D} \eta_{i}^{n+1}\right)+\xi_{i}{ }^{n+1}\right)
\end{aligned}
$$

Initial condition: $\eta_{i}^{1}=\xi_{i}^{1}=0$

Inner boundary condition: $C_{D}\left[\frac{1}{1-\beta_{D} \eta_{1}^{n+1}} \frac{\eta_{1}^{n+1}-\eta_{1}^{n}}{\Delta t}-S \frac{\left(\eta_{2}^{n+1}-\eta_{1}^{n+1}\right)-\left(\eta_{2}^{n}-\eta_{1}^{n}\right)}{\Delta t \Delta x}\right]-\frac{\eta_{2}^{n+1}-\eta_{1}^{n+1}}{\Delta x}=1$

External boundary condition: $\eta_{N}^{n+1}=0$

Finally, we can write the discretized equations in residual form:

$$
\begin{aligned}
& f_{1}= \frac{C_{D} \Delta x}{\Delta t}\left(\eta_{1}^{n+1}-\eta_{1}^{n}\right)-\frac{S C_{D}}{\Delta t}\left(1-\beta_{D} \eta_{1}^{n+1}\right)\left(\eta_{2}^{n+1}-\eta_{1}^{n+1}-\eta_{2}^{n}+\eta_{1}^{n}\right) \\
&-\left(1-\beta_{D} \eta_{1}^{n+1}\right)\left(\eta_{2}^{n+1}-\eta_{1}^{n+1}\right)-\Delta x\left(1-\beta_{D} \eta_{1}^{n+1}\right) \\
& f_{i}=\left(1-\beta_{D} \eta_{i}^{n+1}\right)\left(\eta_{i+1}{ }^{n+1}-2 \eta_{i}{ }^{n+1}+\eta_{i-1}{ }^{n+1}\right) \\
&-\Delta x^{2} e^{2(i-1) \Delta x}\left[\frac{w}{\Delta t}\left(\eta_{i}^{n+1}-\eta_{i}{ }^{n}\right)-\frac{(1-w) \lambda}{1-w+\lambda \Delta t}\left(1-\beta_{D} \eta_{i}^{n+1}\right)\left(\frac{1}{\beta_{D}} \ln \left(1-\beta_{D} \eta_{i}^{n+1}\right)+\xi_{i}{ }^{n}\right)\right] \\
& f_{N}=\eta_{N}^{n+1}
\end{aligned}
$$

Newton-Rapson Method. Newton method is a very efficient algorithm for solving highly nonlinear algebraic equations. First we derive the Jocobian matrix: $J B^{(n)}=\left(\frac{\partial f_{i}}{\partial \eta}\right)^{(n)}$

The resulting JB is a triple diagonal matrix, the three diagonal are $\mathrm{L}, \mathrm{U}$ and $\mathrm{D}$ :

$$
\begin{aligned}
& D(1)=\frac{C_{D} \Delta x}{\Delta t}+\frac{S C_{D}}{\Delta t}\left(1-\beta_{D} \eta_{1}^{n+1}\right)+\frac{S C_{D}}{\Delta t} \beta_{D}\left(\eta_{2}^{n+1}-\eta_{1}^{n+1}-\eta_{2}^{n}+\eta_{1}^{n}\right) \\
& \quad+\beta_{D}\left(\eta_{2}^{n+1}-\eta_{1}^{n+1}\right)+\left(1-\beta_{D} \eta_{1}^{n+1}\right)+\Delta x \beta_{D} \\
& U(1)=-\left(S C_{D} / \Delta t+1\right)\left(1-\beta_{D} \eta_{1}^{n+1}\right) \\
& D(N)=1, L(N)=0 \\
& L(i)=\left(1-\beta_{D} \eta_{i}^{n+1}\right), U(i)=\left(1-\beta_{D} \eta_{i}^{n+1}\right)
\end{aligned}
$$




$$
\begin{aligned}
& D(i)=-\beta_{D}\left(\eta_{i+1}{ }^{n+1}-2 \eta_{i}{ }^{n+1}+\eta_{i-1}{ }^{n+1}\right)-2\left(1-\beta_{D} \eta_{i}{ }^{n+1}\right) \\
& -\Delta x^{2} e^{2(i-1) \Delta x}\left[\frac{w}{\Delta t}+\frac{(1-w) \lambda}{1-w+\lambda \Delta t}\left(\ln \left(1-\beta_{D} \eta_{i}^{n+1}\right)+\beta_{D} \xi_{i}^{n}+1\right)\right]
\end{aligned}
$$

Then iteration can carry on until convergence through the equations [6]:

$$
J B^{(n-1)} \Delta \delta=-f^{(n-1)}, \eta^{n+1}=\Delta \delta+\eta^{n}
$$

\section{Type Curves for Pressure Responses}

Substituting the numerical solutions $\eta$ into the equation of $\varphi_{D f}=-\frac{1}{\beta_{D}} \ln \left(1-\beta_{D} \eta\right)$, we can draw the typical curves of well-testing model in terms of $\varphi_{w D}, \varphi_{w D}^{\prime}$ and $t_{D} / C_{D}$. Then we can analyze the effects of different mechanisms on shapes of pressure responses.

Adsorption. Fig.2(a) shows the log-log plot of pressure responses when Langmuir pressure is 10, 15 and 20. Fig.2(b) shows the curves when Langmuir volume is 10, 3 and 1.

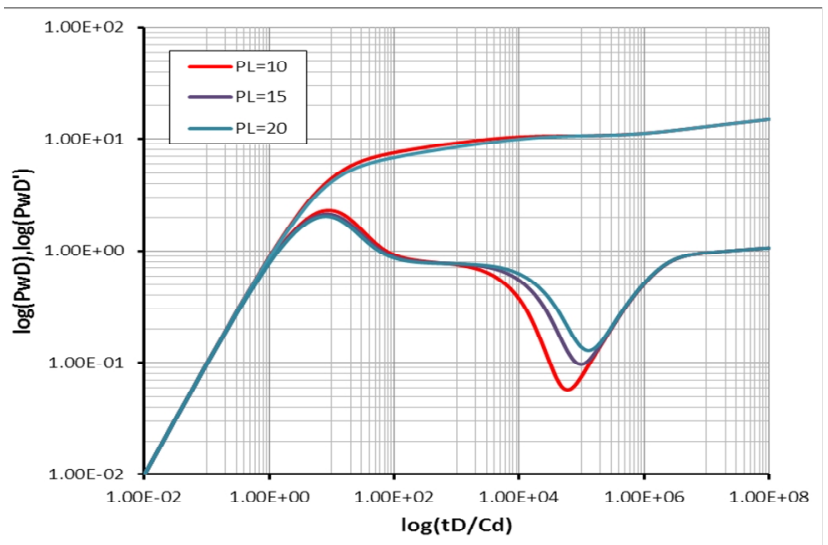

(a)

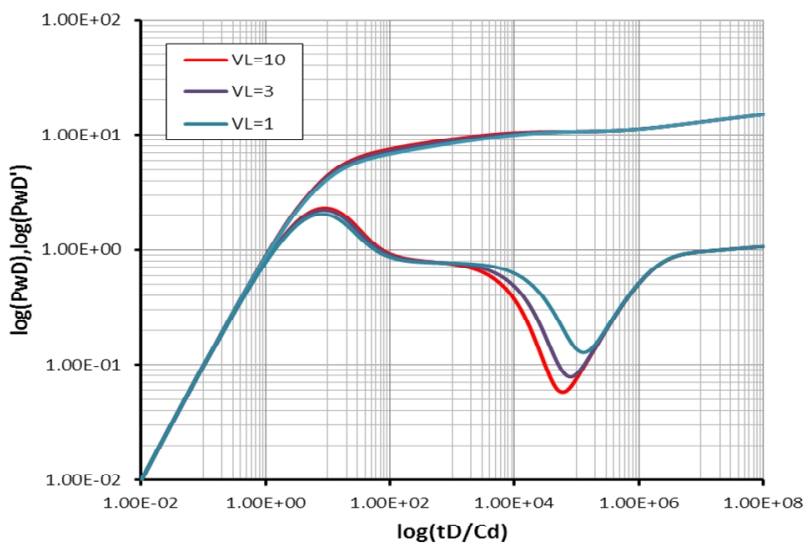

(b)

Fig.2 Influence of Langmuir pressure and volume on pressure transient responses

It can be shown that the higher Langmuir pressure or lower volume, the longer the time needed to let the hollow appear in the stationary interporosity flow section and the lower the minimal value.

Stress Sensitivity. Fig. 3 shows the log-log plot of pressure responses when dimensionless stress sensitive coefficient is $0.01,0.05$ and 0.1 .

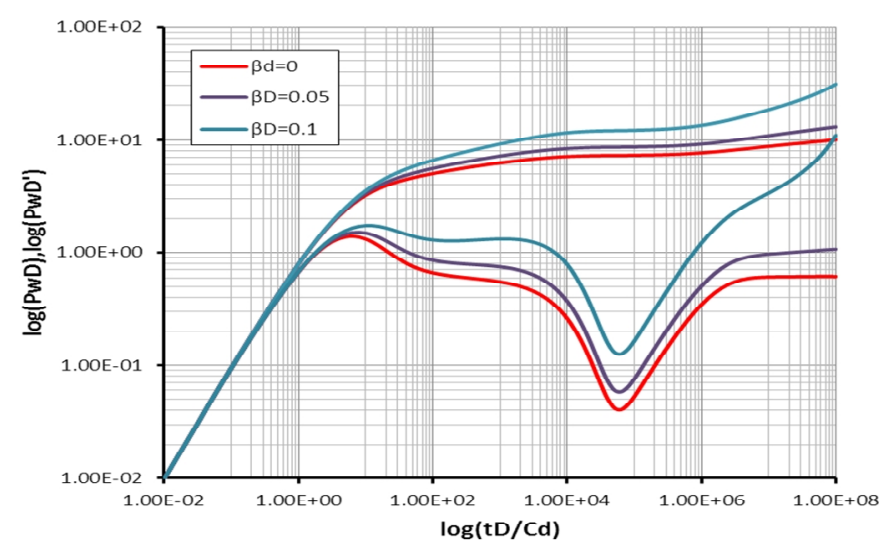

Fig. 3 Influence of stress sensitivity on pressure transient responses

We can see that for boundry flow period, the higher the stress sensitivity, the faster the boundary pressure and pressure derivative curve upward. Analysis also indicates that the greater the stress sensitive coefficient, the greater the pressure and pressure derivative. When it is greater than a certain value, the stationary interporosity-flow section of dual-porosity medium will disappear.

Skin Effect and Wellbore Storage Effect. Fig.4(a) shows the log-log plot of pressure responses when wellbore storage coefficient is $1,0.1$ and 0.01 . Fig.4(b) shows the curves when skin coefficient is 5,2 and 0. 
We can see that the bigger the wellbore storage effect, the greater the peak that occurs in continuous flow period of pressure derivative curve and slower the curve drop. The bigger the skin coefficient, the larger pressure curve and the greater the peak that occurs in continuous flow period.

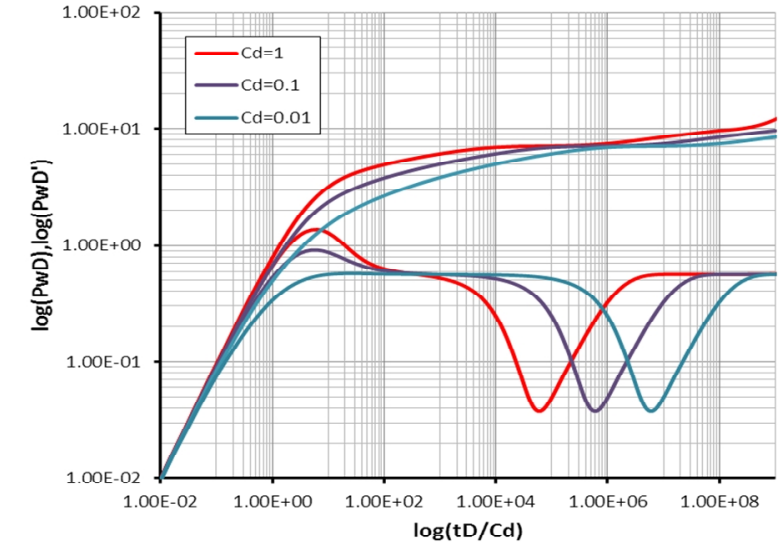

(a)

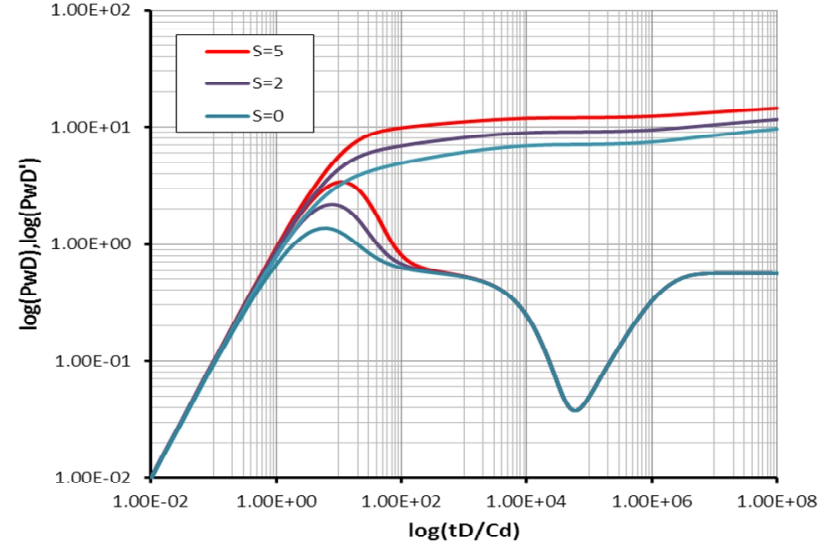

(b)

Fig.4 Influence of skin effect and wellbore storage on pressure transient responses

\section{Conclusions}

In this paper, we present a numerical well-testing model for transient gas flow in naturally fractured shale gas reservoirs. The model incorporates several relevant physical processes, such as adsorption/desorption, pressure-dependent permeability and dual-porosity mechanisiam. The typical curves in terms of dimensionless pseudopressure and time were drawn with different sets of parameters, and the sensitivity analysis was carried out. It can be shown that the nolinear flow mechanisims and conductive, interconnected natural fractures system can substantially influence transient pressure responses of shale gas wells. Therefore well-testing analysis for unconventional gas reservoirs cannot rely entirely on traditional, analytical based approaches and have to be carried out by numerical approach in general.

\section{References}

[1] Wu, Y.S. and Perapon F., "A Unified Mathematical Model for Unconventional Reservoir Simulation," SPE-142884, presented at the SPE EUROPEC Conference, 23-26 May 2011.

[2] Moridis, G.J., Blasingame, T.A., and Freeman, C.M.. Analysis of Mechanisms of Flow in Fractured Tight-Gas and Shale-Gas Reservoirs. Paper SPE 139250 presented at the SPE Latin American \& Caribbean Petroleum Engineering Conference.

[3] Cippolla, C.L., Lolon P., Erdle J.C.. Modeling Well Performance in Shale-Gas Reservoirs, SPE 125532, presented at the 2009 SPE Reservoir Characterization and Simulation Conference.

[4] Langmuir Irving, The constitution and fundamental properties of solids and liquids. Journal of the American Chemical Society 38 (11):2221-2295, 1916.

[5] Wang, F.P., Reed R.M., "Pore Networks and Fluid Flow in Gas Shales," SPE 124253, presented at the 2009 SPE Annual Technical Conference and Exhibition held in New Orleans, Louisiana.

[6] Wu, Y.S., Wang, C., Li J., "Transient Gas Flow in Unconventional Gas Reservoirs”, SPE154448, presented at the EAGE Annual Conference \& Exhibition incorporating SPE Europec held in Copenhagen, Denmark, 4-7 June 2012. 